\title{
CORRESPONDENCE
}

\section{On the care and use of US lab animals}

Some recommendations in the latest Guide for the Care and Use of Laboratory Animals (see go.nature.com/i9y7fr) have stirred up controversy in the research community. As former members of the committee that updated the guide, we would like to comment informally on these objections (see disclaimer).

The guide was released last year by the US National Research Council.

Recommendations may apply to institutions funded by the US Public Health Service, but will, however, be used by the Association for the Assessment and Accreditation of Laboratory Animal Care International, a non-profit organization based in Frederick, Maryland, to evaluate and accredit research institutions.

Because of its many users and settings, the guide is written in broad statements of desired outcomes (performance standards) and is largely devoid of detailed specifications. This enables research institutions to create customized procedures and programmes within the context of the recommendations for high-quality care, from external acquisition or in-house breeding of lab animals to their final disposition.

The latest edition of the guide expands on this approach, partly on the basis of overwhelming support for performance standards by the research community. Despite this, some are urging the National Institutes of Health's Office of Laboratory Animal Welfare (OLAW) not to adopt the eighth edition, spurred by fears over how OLAW will interpret and implement the guide's recommendations. OLAW is currently considering implementation of the guide and is due to issue a position statement soon.

Objections mainly focus on the guide's space recommendations, particularly for breeding rodents. Optimal space requirements depend on factors including strain, sex, age, enrichment and animal psychology. The guide points out the necessity for study and assessment of the space requirements of laboratory species, while leaving previous recommendations essentially unaltered. Emphasis is placed instead on performance standards that are critical for deciding on minimal cage space.

Lobby groups representing commercial rodent vendors (among others) estimate that compliance with this new standard will cost upwards of US\$500 million. But many institutions have already implemented performance standards to prevent overcrowding or are voluntarily using larger breeding cages. Standards for rodent husbandry should not be bound by commercial profitability.

Further research is needed into space and housing requirements and the provision of enrichment, exercise and human contact. We believe that the latest recommendations and a performance-standards approach will eventually help to define the highest welfare standards for research animals. Janet C. Garber on behalf of 13 co-signatories ${ }^{*}$, Garber Consulting, Pinehurst, North Carolina, USA. jgarber@nc.rr.com ${ }^{*}$ See http//dx.doi.org/476152a for a full list of signatories.

Disclaimer: Opinions expressed in this Correspondence are strictly those of the authors, written in their personal capacity. They do not represent the views of colleagues, current or past employers, or any other body.

\section{Rule on papers puts China's PhDs at risk}

To help counter the overemphasis on quantity rather than quality of research publications in China (Nature 475, 267; 2011), many Chinese universities require their $\mathrm{PhD}$ candidates to publish a specified number of papers in high-impact journals before they are awarded their degree.

Although laudable in principle, this requirement puts even more pressure on students. If that is compounded by inadequate guidance and support from mentors, it will encourage more scientific fraud and hamper the development of junior scientists in the long run. Derrick Y. F. Lai McGill University, Montreal, Canada. yukfo.lai@mail.mcgill.ca

\section{Helping hand for genomics in Africa}

We offer a personal example of the benefits of lending African scientists 'a helping hand' (Nature 474, 542; 2011). After a fiveyear relationship, a genomics network partnership was last year officially set up between the J. Craig Venter Institute in San Diego, California, and the University of Limpopo, one of the least resourced universities in South Africa.

The University of Limpopo, with campuses in Polokwane and Pretoria, is the result of a merger between South Africa's University of the North and the Medical University of Southern Africa - previously 'non-white' higher-education facilities as defined by the apartheid policies.

The university is striving to build regional resources and a skill base in genomics to help find scientific solutions for rural Africa. International partnerships, such as the one between our institutions, gives the University of Limpopo's researchers access to current technologies, informatic expertise and training that is unavailable locally.

Together we are seeking practical solutions through educational workshops, student and staff exchanges, improvements in capacity and infrastructure, and equalcontribution projects, while tackling the inevitable challenges as a single unit.

Our experience suggests that 'joining hands' could ultimately prove to be a successful strategy. Vanessa M. Hayes J. Craig Venter Institute, San Diego, California, USA.vhayes@jcvi.org

Philip A. Venter University of Limpopo, Polokwane, South Africa. M. Jeffrey Mphahlele University of Limpopo, Pretoria, South Africa.

\section{Mathematics walks into history}

Mathematician William Rowan Hamilton did indeed hit on the idea of quaternions - a complex-number system extending into four dimensions - while crossing a Dublin bridge in 1843 (Nature 475, 167; 2011).

Yet it was what the Irishman was doing while absorbed in mathematics that gives him the last laugh, for quaternions are used in the clinical and laboratory analysis of bodily motion, notably of gait. So what was Hamilton doing? Walking into history.

John Hart Oxford Brookes University, Oxford, UK. johnhart1@btconnect.com

\section{CONTRIBUTIONS}

Correspondence may be sent to correspondence@ nature.com after consulting the author guidelines at go.nature.com/cmchno 